Jennifer Yeung, MD

Cécile Cauquil, MD

Guillaume Saliou, MD,

$\mathrm{PhD}$

Ghaida Nasser, MD

Sophio Rostomashvili, MD

David Adams, MD, PhD

Marie Théaudin, MD, $\mathrm{PhD}$

\section{VARICELLA-ZOSTER VIRUS ACUTE MYELITIS IN A PATIENT WITH MS TREATED WITH NATALIZUMAB}

We report a case of varicella-zoster virus (VZV) myelitis in a woman with relapsing-remitting multiple sclerosis (RRMS) receiving natalizumab, a humanized monoclonal antibody that induces an immunosuppression localized to the CNS.

Case report. A 32-year-old woman was treated with natalizumab for highly active RRMS. After the fourth infusion, she complained of a right radicular pain in a $\mathrm{L} 5 / \mathrm{S} 1$ territory. A few days later, the neurologic examination disclosed a distal weakness (3/5 Medical Research Council [MRC]) in the right leg with signs of pyramidal irritation suggesting a spinal cord relapse. The spinal cord MRI disclosed focal cervical and dorsal T2 hyperintensities and a T2 hypersignal in the conus region with contrast enhancement (figure). A spinal cord relapse was considered. High doses of IV methylprednisolone were initiated for 3 days. Two weeks later, the patient deteriorated, with an increase of leg weakness (2/5 MRC) and bladder dysfunction. She received another series of IV methylprednisolone for 3 days, without improvement. Because of this unusual evolution, we performed a lumbar puncture, which revealed a lymphocytic pleocytosis (18 elements), normal proteins, and glycorrhachia. VZV DNA detected by PCR amplification was positive in CSF. Cytomegalovirus and herpes simplex virus (HSV) PCR in the CSF were negative. HIV screening was negative. VZV immunoglobulin $\mathrm{G}$ in the blood was positive before this acute episode (tested in January 2012). No skin rash was noted or reported by the patient. A second spinal cord MRI showed progression of lesion size, persistent contrast enhancement in the conus region, and new contrast enhancements in dorsal lesions (figure). Acute VZV myelitis was diagnosed. The patient was treated with IV acyclovir $10 \mathrm{mg} / \mathrm{kg} / 8 \mathrm{~h}$ for 3 weeks and then switched to valacyclovir, which resulted in a clinical improvement (4/5 MRC in right lower limb and recovery of subnormal bladder function but persistent sensory loss in the left lower limb). CSF at 1 month was normal (4 elements, negative VZV PCR). Spinal cord MRI at 2 months was clearly improved (figure). Natalizumab was discontinued.

Discussion. If cases of HSV encephalitis and meningitis have been previously reported, ${ }^{1,2}$ no previous case of
Clinical/Scientific Notes

VZV infection in patients receiving natalizumab therapy has been described, to our knowledge. We did not find cases of spinal cord viral infections in patients receiving natalizumab among published literature. However, VZV infections can occur with other monoclonal antibodies therapy, such as anti-tumor necrosis factor- $\alpha$ therapy. Indeed, in 2004, Keystone et al. ${ }^{4}$ reported a case of herpes zoster encephalitis in a patient with rheumatoid arthritis receiving long-term adalimumab associated with methotrexate. ${ }^{3}$ In 2010, a second case of VZV encephalitis was described in a patient with psoriatic arthritis who was treated by adalimumab for 1.5 years. VZV encephalitis is a rare and severe clinical manifestation due to a primitive viral infection or a reactivation of latent VZV. We believe that our patient had a VZV reactivation favored by natalizumab rather than a new infection. Indeed, she was immunized against VZV (presence of immunoglobulin G anti-VZV before the onset of natalizumab treatment and chickenpox in childhood) and there was no other cause than natalizumab for immune deficiency.

It may be difficult at the onset of symptoms to differentiate a CNS viral infection from a relapse, in particular when the site of infection is the spinal cord. Spinal cord MRI is useful when clinical evolution is unusual for multiple sclerosis relapse, as well as lumbar puncture, which should be performed as early as possible. Targeting people at high risk to develop these viral infections on natalizumab therapy is a great challenge. This could allow applying easy preventive measures if necessary (such as acyclovir daily intake, which can prevent VZV infection in multiple myeloma patients receiving bortezomib therapy). ${ }^{5}$ Neurologists prescribing natalizumab should not underestimate the risk of herpes and zoster infection because early diagnosis and treatment are the keys to a better prognosis.

From AP-HP (J.Y., C.C., G.S., G.N., S.R., D.A., M.T.), Hôpital Bicêtre; UMR8081 CNRS (M.T.) and UMR788 INSERM (D.A.), Le Kremlin-Bicêtre, France.

Author contributions: Dr. Yeung: drafting the manuscript for content, including medical writing for content, analysis or interpretation of data, acquisition of data. Dr. Cauquil: revising the manuscript for content, including medical writing for content, analysis or interpretation of data. Dr. Saliou: revising the manuscript for content, including medical writing for content. Dr. Nasser: revising the manuscript for content, including medical writing for content. Dr. Rostomashvili: revising the manuscript for content, including medical writing for content, acquisition of data. Pr. Adams: revising the 

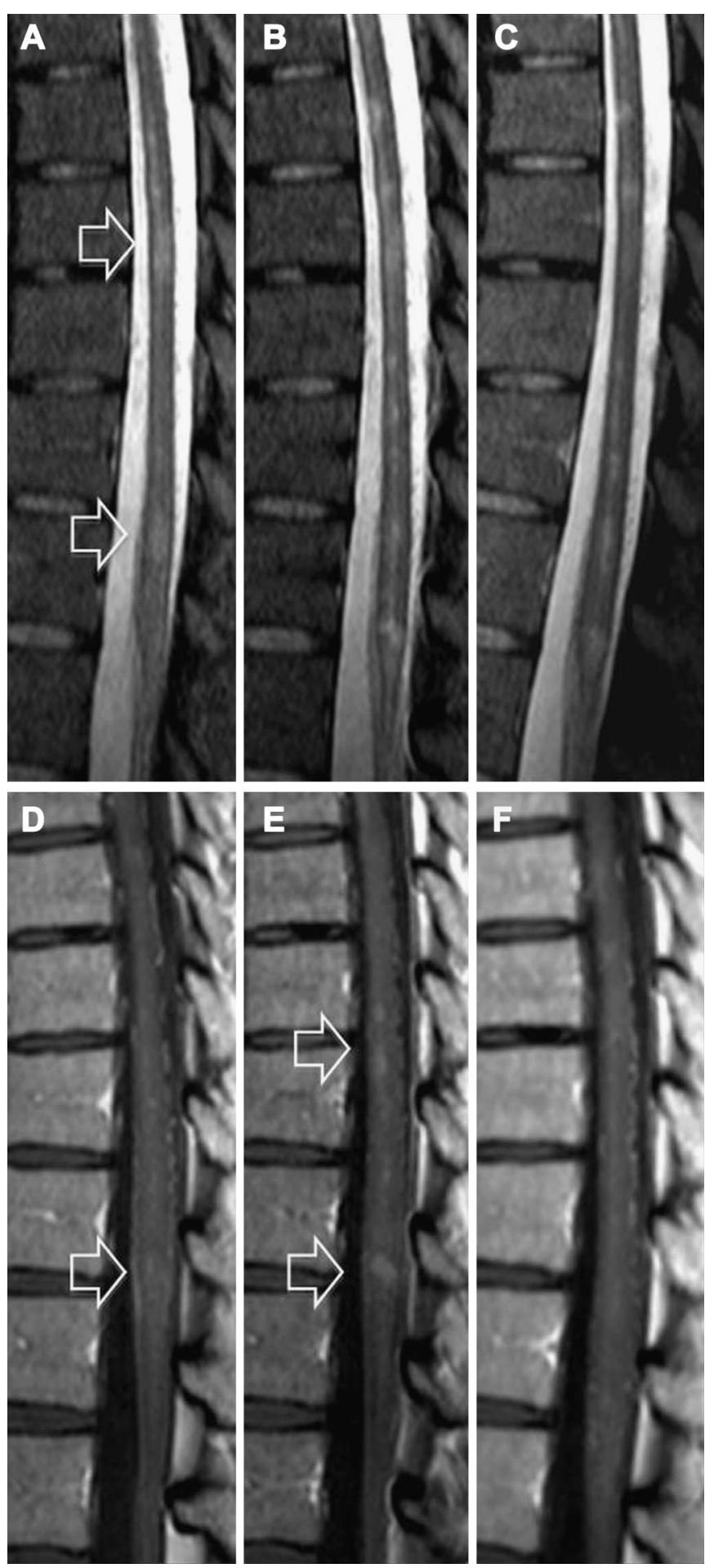

Sagittal STIR (A-C) and T1-weighted sequences after gadolinium (D-F) at the acute stage (A and $D)$, at day 15 (B and $E$ ), and at 2 months (C and F). The initial MRI (A, D) shows dorsal hyperintensities (A: empty arrows) with slight enhancement of the lower one (D: empty arrow), leading to a diagnosis of multiple sclerosis relapse. The second MRI (after administration of methylprednisolone) (B, E) shows increased size of previous hyperintensities and new hyperintensities in STIR (B) and new gadolinium enhancements in T1-weighted sequences (E: empty arrows). At this time, the diagnosis of varicella-zoster virus myelitis was made and acyclovir treatment was initiated. The final MRI (after acyclovir/valacyclovir treatment) shows decreased size of hyperintensities in STIR (C) and a marked decrease of contrast enhancements (F). manuscript for content, including medical writing for content. Dr. Théaudin: revising the manuscript for content, including medical writing for content, analysis or interpretation of data, acquisition of data, study supervision or coordination.

Study funding: No targeted funding reported.

Disclosure: J. Yeung reports no disclosures. C. Cauquil received funding for medical congresses from LFB. G. Saliou, G. Nasser, and $S$. Rostomashvili report no disclosures. D. Adams serves as a consultant in the EMA and received funding for a medical congress from Baxter and Bayer Schering Pharma. M. Théaudin received funding for medical congresses from Biogen, Bayer Schering Pharma, Teva, and LFB. Go to Neurology.org for full disclosures.

Received November 8, 2012. Accepted in final form January 19, 2013.

Correspondence to Dr. Théaudin: marie.theaudin@bct.aphp.fr

\section{2013 American Academy of Neurology}

1. Seiguer Shenoy E, Mylonakis E, Hurtado RM, Venna N. Natalizumab and HSV meningitis. J Neurovirol 2011;17: 288-290.

2. Kwiatkowski A, Gallois J, Bilbault N, Calais G, Mackowiak A, Hautecoeur P. Herpes encephalitis during natalizumab treatment in multiple sclerosis. Mult Scler 2012;18:909-911.

3. Keystone EC, Kavanaugh AF, Sharp JT, et al. Radiographic, clinical, and functional outcomes of treatment with adalimumab (a human anti-tumor necrosis factor monoclonal antibody) in patients with active rheumatoid arthritis receiving concomitant methotrexate therapy: a randomized, placebo-controlled, 52-week trial. Arthritis Rheum 2004;50: 1400-1410.

4. Buccoliero G, Loreno G, Romanelli C, Looperfido P, Resta F. Varicella zoster virus encephalitis during treatment with anti-tumor necrosis factor $\alpha$ agent in a psoriatic arthritis patient. New Microb 2010;33:271-274.

5. Vickrey E, Allen S, Mehta J, Singhal S. Acyclovir to prevent reactivation of varicella zoster virus (herpes zoster) in multiple myeloma patients receiving bortezomib therapy. Cancer 2009;115:229-232. 